Honam Mathematical J. 35 (2013), No. 1, pp. 101-107

http://dx.doi.org/10.5831/HMJ.2013.35.1.101

\title{
NOTE ON CONVERGENCE OF EULER'S GAMMA FUNCTION
}

\author{
Junesang Choi
}

\begin{abstract}
The Gamma function $\Gamma$ which was first introduced by Euler in 1730 has played a very important role in many branches of mathematics, especially, in the theory of special functions, and has been introduced in most of calculus textbooks. In this note, our major aim is to explain the convergence of the Euler's Gamma function expressed as an improper integral by using some elementary properties and a fundamental axiom holding on the set of real numbers $\mathbb{R}$, in a detailed and instructive manner. A brief history and origin of the Gamma function is also considered.
\end{abstract}

\section{Introduction and Preliminaries}

Integration has been introduced and taught from high schools. In fact, from this point up to the various investigations having been conducted by experienced researchers, integration has played a very important role. Thus a lot of handbooks each of which contains a remarkably large number of integral formulas have been published (see, for example, [3]). The Gamma function $\Gamma$ which was first introduced by Euler in 1730 has played a very important role in many branches of mathematics, especially, in the theory of special functions, and has been introduced in most of calculus textbooks (see, for example, [7, p. 442]). In this note, our major aim is to explain the convergence of the Euler's Gamma function $\Gamma$ expressed as an improper integral by using some elementary properties and a fundamental axiom holding on the set of real numbers $\mathbb{R}$, in a detailed and instructive manner. A brief history and origin of the Gamma function is also considered. To do this, we begin by recalling the

Received February 15, 2013. Accepted February 21, 2013.

2010 Mathematics Subject Classification. Primary 33B15, 26A42; Secondary $26 \mathrm{~A} 03$.

Key words and phrases. Gamma function; Riemann integral; Improper integral; Completeness axiom; Approximation property for suprema; Archimedean principle. 
completeness axiom which is one of the four axioms being received as true fundamental mathematical facts holding on the set of real numbers $\mathbb{R}$.

Completeness Axiom (see, for example, [8, p. 18]). If $E$ is a nonempty subset of $\mathbb{R}$ that is bounded above, then $E$ has a finite supremum.

This completeness axiom has often been used and involved, directly or indirectly, in investigating and verifying some properties and theorems regarding $\mathbb{R}$. We recall a known property and a well-known principle related to this completeness axiom.

Approximation Property for Suprema (see, for example, [8, p. 17]). If $E$ has a finite supremum and $\varepsilon>0$ is any positive number, then there is a point $a \in E$ such that

$$
\sup E-\varepsilon<a \leq \sup E .
$$

Archimedean Principle (see, for example, [8, p. 18]). The set of natural numbers $\mathbb{N}:=\{1,2,3, \ldots\}$ is not bounded above in $\mathbb{R}$. That is, for every $x \in \mathbb{R}$, there is $n \in \mathbb{N}$ such that $x<n$.

Riemann Integrals and Improper Integrals. Here we introduce Riemann integrals and improper integrals very shortly in order to point out the difference between these two integrals. We give a very brief account of the Riemann integral and the improper integral of a real-valued function $f$ defined on an interval of $\mathbb{R}$. For a detailed definition of the Riemann integral $\int_{a}^{b} f(x) d x$ and its properties, the reader may be referred to elementary analysis textbooks (see, for example, $[8$, Chapter 5]). To define the Riemann integral $\int_{a}^{b} f(x) d x$, the following two conditions are at least satisfied:

(i) The interval $[a, b]$ is closed and bounded.

(ii) The function $f:[a, b] \rightarrow \mathbb{R}$ is bounded on the closed bounded interval $[a, b]$.

Let us consider a function $f: E \rightarrow \mathbb{R}(E$ is an interval in $\mathbb{R})$ which does not satisfy the above two necessary conditions (i) and (ii). That is, $E=$ $[a, b),(a, b],(a, b),(-\infty, b],[a, \infty),(-\infty, b),(a, \infty),(-\infty, \infty)=\mathbb{R}$, or $f$ is not a bounded function. In this case, we can define the improper integral of (for example) a function $f:(a, b) \rightarrow \mathbb{R}$ if the following two conditions are satisfied: 
(a) The function $f:(a, b) \rightarrow \mathbb{R}$ is Riemman integrable on any closed bounded interval $[c, d] \subset(a, b)$.

(b) Then the following limit exits:

$$
\lim _{c \rightarrow a} \lim _{d \rightarrow b} \int_{c}^{d} f(x) d x=\lim _{d \rightarrow b} \lim _{c \rightarrow a} \int_{c}^{d} f(x) d x .
$$

If a function $f:(a, b) \rightarrow \mathbb{R}$ satisfies (a) and (b), $f$ is said to be $i m$ properly integrable on $(a, b)$ and its improper integral is still denoted by $\int_{a}^{b} f(x) d x$ and defined by

$$
\int_{a}^{b} f(x) d x:=\lim _{c \rightarrow a} \lim _{d \rightarrow b} \int_{c}^{d} f(x) d x .
$$

\section{Gamma Function}

Leonhard Euler (1707-1783) introduced and defined the Gamma function $\Gamma$ by

$$
\Gamma(x):=\int_{0}^{\infty} e^{-t} t^{x-1} d t \quad(x>0) .
$$

In the earlier part of the eighteenth century, there were two main streams in the mathematical research: Interpolation and Integration. The familiar factorial function

$$
f(n)=n !:=n \cdot(n-1) \cdots 2 \cdot 1 \quad\left(n \in \mathbb{N}_{0}\right) \quad \text { and } \quad 0 !:=1
$$

is a real-valued function defined on $\mathbb{N}_{0}:=\mathbb{N} \cup\{0\}$. At the Euler's time, a natural question had been posed: How can we interpolate the factorial function $f(n)=n$ !? That is, how can we define this factorial function $f(n)=n$ ! on the set of real numbers between two consecutive nonnegative integers so that the resulting interpolated function can have some useful mathematical properties? In fact, it is seen that a few minutes' thought can give us an innumerable number of ways to interpolate this factorial function $f(n)=n$ ! (see, for example, [4]). Yet it is important for us to find a useful interpolation in a mathematical sense. Indeed, a solution of this interpolation can be traced back to two letters from Leonhard Euler (1707-1783) to Christian Goldbach (1690-1764), just as a simple desire to extend factorials to values between the integers. The first letter (dated October 13, 1729) dealt with the interpolation problem, while the second letter (dated January 8, 1730) dealt with integration and tied the two together. Eventually Euler defined the Gamma function $\Gamma$ as in (2.1). The present notation $\Gamma$ was introduced 
by Adrien-Marie Legendre (1752-1833) in 1814. The Gamma function in $(2.1)$ can be naturally extended on the set of complex numbers $\mathbb{C}$ as follows:

$$
\Gamma(z)=\int_{0}^{\infty} e^{-t} t^{z-1} d t \quad(z \in \mathbb{C} ; \Re(z)>0) .
$$

It is surprising that this Euler's Gamma function $\Gamma$ has a large number of interesting and useful properties (see, for example, [5, Section 1.1] and [6, Section 1.1]). Among them, the following easily derivable value and relation are important in view of its birth and application:

$$
\Gamma(1)=1 \quad \text { and } \quad \Gamma(x+1)=x \Gamma(x) \quad\left(x \in \mathbb{R}^{+}\right),
$$

where $\mathbb{R}^{+}$denotes the set of positive real numbers. It is easy to find from (2.3) that

$$
\Gamma(n+1)=n ! \quad\left(n \in \mathbb{N}_{0}\right),
$$

which shows that this Gamma function in (2.1) is a solution of the interpolation problem of the factorial function $f(n)=n$ ! on the set of real numbers $\mathbb{R}$. In fact, since the time of Euler, this Gamma function has turned out to be a simplest and most important special function. Therefore knowledge of the Gamma function's properties is a prerequisite for the study of many other special functions. The Gamma function arises in many areas of mathematics. In [4], Davis gave a good and elegant exposition for the origin, history, and development process of this Gamma function. There are several useful other expressions for this Gamma function, (2.1) of which has been introduced in calculus, elementary real and complex analysis textbooks (see, for example, [7, p. 442], [8, pp. 514-522] and [2, pp. 235-242]).

\section{Convergence of the improper integral (2.1)}

Here we will prove the convergence of the improper integral (2.1) under the given condition $x>0$. That is, we show that the improper integral (2.1) is really a finite fixed real value by using several elementary mathematical properties and an axiom.

It is noted that the improper integral (2.1) can be separated into two parts, in view of the definition of improper integral (1.1), each of which 
can be expressed as follows:

$$
\begin{aligned}
\Gamma(x) & =\int_{0}^{1} e^{-t} t^{x-1} d t+\int_{1}^{\infty} e^{-t} t^{x-1} d t \\
& =\lim _{\delta \downarrow 0} \int_{\delta}^{1} e^{-t} t^{x-1} d t+\lim _{\eta \uparrow \infty} \int_{1}^{\eta} e^{-t} t^{x-1} d t .
\end{aligned}
$$

Before going further, it is remarked that, for every $\delta$ with $0<\delta \leq 1$, the integrand $e^{-t} t^{x-1}$ is a continuous function of the variable $t$ on the closed bounded interval $[\delta, 1]$. Therefore, in view of a well-known property for Riemann integral (see, for example, [8, p. 134, Theorem 5.10]: Every continuous real-valued function defined on a closed bounded interval is Riemann integrable), for every $\delta$ with $0<\delta \leq 1$, the Riemann integral $\int_{\delta}^{1} e^{-t} t^{x-1} d t$ exists. Likewise, for every $\eta$ with $\eta \geq 1$, the Riemann integral $\int_{1}^{\eta} e^{-t} t^{x-1} d t$ also exists.

Therefore, to prove the convergence of the improper integral (2.1), it is enough to show that the two limits given in the second equality in (3.1) exist as a finite real value. For convenience, we will treat with the existence of the two limits separately.

Case 1. The existence of $\lim _{\delta \downarrow 0} \int_{\delta}^{1} e^{-t} t^{x-1} d t$.

Since the integrand $e^{-t} t^{x-1}$ is positive for the variable $t$ and $e^{-t} \leq 1$ on the closed bounded interval $[\delta, 1]$, for every $\delta$ with $0<\delta \leq 1$ and each $x \in \mathbb{R}^{+}$, the following inequalities hold true:

$$
0 \leq \int_{\delta}^{1} e^{-t} t^{x-1} d t \leq \int_{\delta}^{1} t^{x-1} d t=\left.\frac{t^{x}}{x}\right|_{\delta} ^{1}=\frac{1}{x}-\frac{\delta^{x}}{x}<\frac{1}{x}
$$

Now, for $x>0$ being fixed, the following set

$$
E:=\left\{\int_{\delta}^{1} e^{-t} t^{x-1} d t \mid 0<\delta \leq 1\right\}
$$

is easily seen to be a nonempty subset of $\mathbb{R}$ which is bounded above by $1 / x$. Therefore, by the completeness axiom, $\sup E$ exists as a finite real number. Here, we show that $\lim _{\delta \downarrow 0} \int_{\delta}^{1} e^{-t} t^{x-1} d t=\sup E$. This will complete the proof in this case. Indeed, for each given $\varepsilon>0$, by the approximation property for suprema, there exists $\delta_{\varepsilon}$ depending on the $\varepsilon$ such that $0<\delta_{\varepsilon} \leq 1$ and

$$
\sup E-\varepsilon<\int_{\delta_{\varepsilon}}^{1} e^{-t} t^{x-1} d t \leq \sup E .
$$


For every $\delta$ with $0<\delta \leq 1$, since the integrand $e^{-t} t^{x-1}$ is positive on the closed bounded interval $[\delta, 1]$, as $\delta \downarrow 0$, the integral $\int_{\delta}^{1} e^{-t} t^{x-1} d t$ increases. So, for every $\delta$ with $0<\delta \leq \delta_{\varepsilon}$, the following inequalities hold true:

$$
\sup E-\varepsilon<\int_{\delta_{\varepsilon}}^{1} e^{-t} t^{x-1} d t \leq \int_{\delta}^{1} e^{-t} t^{x-1} d t \leq \sup E<\sup E+\varepsilon,
$$

which is equivalently rewritten as

$$
\left|\int_{\delta}^{1} e^{-t} t^{x-1} d t-\sup E\right|<\varepsilon
$$

Hence it follows from the definition of limit that

$$
\lim _{\delta \downarrow} \int_{\delta}^{1} e^{-t} t^{x-1} d t=\sup E .
$$

Case 2. The existence of $\lim _{\eta \uparrow \infty} \int_{1}^{\eta} e^{-t} t^{x-1} d t$.

We begin by showing the following inequality:

$$
e^{t}>\frac{t^{n}}{n !} \quad\left(n \in \mathbb{N}_{0} ; t \in \mathbb{R}^{+}\right) .
$$

An easy method of the proofs of the inequality (3.2) is enough to recall the following Maclaurin series for $e^{t}$ :

$$
e^{t}=\sum_{n=0}^{\infty} \frac{t^{n}}{n !} \quad(-\infty<t<\infty) .
$$

Therefore, for every $t>0$ and each $n \in \mathbb{N}_{0}$, the following inequality holds true:

$$
e^{-t} t^{x-1}<\frac{n !}{t^{n+1-x}}
$$

Now let $x \in \mathbb{R}^{+}$be fixed. For this fixed $x>0$, by the Archimedean principle, we can choose $n \in \mathbb{N}$ so large that $n>x$. We thus find that, for this fixed $x>0$ and such chosen $n \in \mathbb{N}$, the following inequalities hold true:

$$
\int_{1}^{\eta} e^{-t} t^{x-1} d t<n ! \int_{1}^{\eta} \frac{d t}{t^{n+1-x}}=\frac{n !}{n-x}\left(1-\eta^{-n+x}\right)<\frac{n !}{n-x} .
$$

Now, for $x>0$ being fixed, the following set

$$
F:=\left\{\int_{1}^{\eta} e^{-t} t^{x-1} d t \mid \eta \geq 1\right\}
$$


is seen to be a nonempty subset of $\mathbb{R}$ which is bounded above by $n ! / n-x$. So it follows from the completeness axiom that $\sup F$ exists as a finite real number. From now on, by using the same argument as in Case 1, it is easy to show that

$$
\lim _{\eta \uparrow \infty} \int_{1}^{\eta} e^{-t} t^{x-1} d t=\sup F
$$

We conclude that, in view of Case 1 and Case 2 , for each fixed $x \in \mathbb{R}^{+}$, the improper integral in (2.1) is shown to be truly a fixed real number.

\section{References}

[1] E. Artin, The Gamma Function, Holt, Rinehart and Winston, New York, Chicago, San Francisco, Toronto and London, 1964.

[2] J. Bak and D. J. Newman, Complex Analysis, second edition, Springer-Verlag, New York, 1997,

[3] Y. A. Brychkov, Handbook of Special Functions, Derivatives, Integrals, Series and Other Formulas, Taylor \& Francis Group, LLC, 2008.

[4] P. J. Davis, Leonhard Euler's integral; a historical profile of the Gamma function, Amer. Math. Monthly 66 (1959), 849-869.

[5] H. M. Srivastava and J. Choi, Series Associated with the Zeta and Related Functions, Kluwer Academic Publishers, Dordrecht, Boston and London, 2001.

[6] H. M. Srivastava and J. Choi, Zeta and q-Zeta Functions and Associated Series and Integrals, Elsevier Science Publishers, Amsterdam, London and New York, 2012.

[7] D. Varberg and E. J. Purcell, Calculus with Analytic Geometry, sixth edition, Prentice Hall, Englewood Cliffs, New Jersey 07632, 1992.

[8] W. R. Wade, An Intorduction to Analysis, fouth edition, Pearson Prentice Hall; Pearson Education, Inc., 2010.

[9] E. T. Whittaker and G. N. Watson, A Course of Modern Analysis: An Introduction to the General Theory of Infinite Processes and of Analytic Functions; With an Account of the Principal Transcendental Functions, fourth edition, Cambridge University Press, Cambridge, London and New York, 1963.

Junesang Choi

Department of Mathematics, Dongguk University, Gyeongju 780-714, Republic of Korea.

E-Mail: junesang@mail.dongguk.ac.kr 